\title{
Cooperative role of RanBP9 and P73 in mitochondria-mediated apoptosis
}

\author{
T Liu', SE Roh ${ }^{1}$, JA Woo ${ }^{1}, \mathrm{H} \mathrm{Ryu}^{1,2}$ and DE Kang ${ }^{*, 1,3}$
}

Mitochondrial dysfunction and synaptic damage are critical early features of Alzheimer's disease (AD) associated with amyloid $\beta$ $(A \beta)$ and $\tau$. We previously reported that the scaffolding protein RanBP9, which is overall increased in AD, simultaneously promotes A $\beta$ generation and focal adhesion disruption by accelerating the endocytosis of APP and $\beta 1$-integrin, respectively. Moreover, RanBP9 induces neurodegeneration in vitro and in vivo and mediates A $\beta$-induced neurotoxicity. However, little is known regarding the mechanisms underlying such neurotoxic processes. Here, we show that RanBP9 induces the loss of mitochondrial membrane potential and increase in mitochondrial superoxides associated with decrease in Bcl-2, increase in Bax protein and oligomerization, fragmentation of mitochondria, and cytochrome $c$ release. RanBP9-induced neurotoxic changes are significantly prevented by the mitochondrial fission inhibitor Mdivi-1 and by classical inhibitors of the mitochondrial apoptosis, XIAP, Bcl-2, and Bcl-xl. RanBP9 physically interacts with the tumor suppressor p73 and increases endogenous p73 $\alpha$ levels at both transcriptional and post-translational levels;moreover, the knockdown of endogenous p73 by siRNA effectively blocks RanBP9 and AB1-42-induced mitochondria-mediated cell death. Conversely, siRNA knockdown of endogenous RanBP9 also suppresses p73-induced apoptosis, suggesting that RanBP9 and p73 have cooperative roles in inducing cell death. Taken together, these finding implicate the RanBP9/p73 complex in mitochondria-mediated apoptosis in addition to its role in enhancing $A \beta$ generation.

Cell Death and Disease (2013) 4, e476; doi:10.1038/cddis.2012.203; published online 24 January 2013

Subject Category: Cancer

The accumulations of amyloid $\beta(\mathrm{A} \beta)$ peptide and hyperphosphorylated $\tau$ are the major pathological hallmarks of Alzheimer's disease (AD). Mounting evidence clearly indicates that mitochondrial dysfunction is a critical early component of $A D$ and related neurodegenerative disorders. ${ }^{1-3}$ Specifically, $\mathrm{A} \beta$ contributes to neurodegeneration through activation of intrinsic apoptotic pathways involving mitochondria. $^{4-6}$ It has been reported that $A \beta$ localizes to mitochondrial membrane and impairs mitochondrial functions through interacting with mitochondrial proteins, disrupting electron-transport chain and increasing mitochondrial ROS products. ${ }^{7-9}$ A recent study also demonstrated early deficits in synaptic mitochondria, $A \beta$ accumulation within mitochondria prior to extracellular $\mathrm{A} \beta$ deposition, and impaired axonal transport of mitochondria in mutant APP transgenic mice. ${ }^{10}$

Mitochondria-mediated apoptosis is the best known intrinsic apoptotic pathway. Impaired mitochondrial function is associated with the aging process and prevalent agerelated diseases including AD. ${ }^{11,12}$ Conversely, perturbation in mitochondria-mediated apoptosis has a critical role in oncogenic processes and downstream effects of tumor suppressor proteins such as p53 and p73. Cellular stress from DNA damage, loss of cell survival factors or defective cell cycle promotes the accumulation of pro-apoptotic proteins, such as Bax, Bak, Noxa, and puma. ${ }^{13}$ Meanwhile, antiapoptotic proteins such as $\mathrm{Bcl}-2$ and $\mathrm{Bcl}-\mathrm{xl}$ prevent apoptosis by inhibiting the action of pro-apoptotic proteins. ${ }^{14,15}$ Accordingly, when the balance of activity between pro- and antiapoptotic members is upset, the permeability of mitochondrial membrane is lost and mitochondrial reactive oxygen species (ROS) is induced. ${ }^{16,17}$ Apoptogenic proteins like cytochrome $c$ or apoptotic inducing factors are then released to the cytosol, which activate pro-caspases to induce apoptosis. ${ }^{18}$

We recently demonstrated that the scaffolding protein RanBP9 interacts with the cytoplasmic tails of LRP, APP and BACE1, and functions as a scaffold upon which APP is brought together with BACE1 and LRP. Such interactions of RanBP9 promote the endocytosis of APP and strongly increase BACE1 cleavage of APP to generate $A \beta$ in cultured cells and in vivo. ${ }^{19,20}$ In addition, a $60-\mathrm{kD}$ proteolytic fragment of RanBP9 is robustly increased in the brains of AD patients, and this fragment strongly potentiates $\mathrm{A} \beta$ generation via

\footnotetext{
${ }^{1}$ WCU-Neurocytomics Program, Seoul National University College of Medicine, Seoul, Korea; ${ }^{2}$ Department of Neurology, Boston University School of Medicine, Boston, MA, USA and ${ }^{3}$ Department of Molecular Medicine, USF Health College of Medicine, Byrd Alzheimer's Institute, Tampa, FL, USA

${ }^{*}$ Corresponding author: DE Kang, Department of Molecular Medicine, USF Health College of Medicine, Byrd Alzheimer's Institute, Tampa, FL 33613, USA. Tel: + 858519 7081; Fax: + 858519 7081; E-mail: dkang@health.usf.edu

Keywords: amyloid; apoptosis; RanBPM; p73; cofilin; mitochondria

Abbreviations: A $\beta$, Amyloid $\beta$ peptide; AD, Alzheimer's disease; APP, Amyloid precursor protein; BACE1, $\beta$ site app cleaving enzyme 1; DIV, Days in vitro; DRP1, Dynamin-related protein 1; EGFP, Enhanced Green Fluorescent Protein; FACS, Fluorescence activated cell sorter; FAD, Familial Alzheimer's disease; GAPDH, Glyceraldehyde 3-phosphate dehydrogenase; LDH, Lactate dehydrogenase; LRP, Low density lipoprotein receptor-related protein; MMP, Mitochondrial membrane potential; PI, Propidium iodide; RanBP9, Ran-binding protein 9; ROS, Reactive oxygen species; RT-qPCR, Reverse transcription-quantitative polymerase chain reaction; TRAIL, Tumor necrosis factor-related apoptosis inducing ligand; XIAP, X-linked inhibitor of apoptosis protein

Received 21.5.12; revised 13.10.12; accepted 17.10.12; Edited by D Bano
} 
BACE1 processing of APP. ${ }^{21}$ We also recently demonstrated that RanBP9 functions to inhibit cell adhesion by accelerating the endocytosis of $\beta 1$-integrin complexes ${ }^{22}$ and promotes apoptosis at least in part by activating the actin- and mitochondria-associated protein cofilin. ${ }^{23}$ Indeed, RanBP9 protein levels were increased four-fold in mutant APP transgenic mice, and RanBP9 transgenic mice demonstrated significantly increased synapse loss, neurodegeneration, gliosis and spatial memory deficits, ${ }^{23}$ demonstrating a link to $A D$ and neurodegeneration. ${ }^{24}$

Consistent with these observations, a recent study showed that RanBP9 also exerts pro-apoptotic activity in DNA damage-induced apoptosis by regulating $\mathrm{Bax}$ and $\mathrm{Bcl}-2$ protein levels in mitochondria. ${ }^{13}$ It has also been reported that the physical association of RanBP9 with the C-terminus of the tumor suppressor protein $p 73 \alpha$ modulates exogenously expressed $p 73 \alpha$ levels and nuclear translocation of RanBP9. ${ }^{25}$ Moreover, it has been shown that $p 73$ can induce apoptosis via nuclear and non-nuclear pathways, the latter involving direct translocation into mitochondria. ${ }^{26}$ However, the mechanism of RanBP9-induced apoptosis, the involvement of mitochondria in such process, and the functional role of the RanBP9/p73 complex are not well understood. In this study, we found that RanBP9 together with p73 induce aberrant changes in mitochondria (MMP, superoxide levels, apoptotic proteins \& fission) and induce apoptosis that depend on their cooperative actions. Such results implicate the critical role of the RanBP9/p73 pathway in the regulation of mitochondria-mediated apoptosis during neurodegenerative processes.

\section{Results}

Excessive RanBP9 induces mitochondrial membrane permeability and promotes apoptosis in mouse hippocampal HT22 cells. It has been reported that overexpression of RanBP9 can increase the activation of caspases and induce cell death in Hela cells. ${ }^{13}$ Consistent with this observation, we also showed that RanBP9 induces neurodegeneration in vivo and mediates $\mathrm{A} \beta$-induced toxicity. ${ }^{23}$ In HT22 cells transiently transfected with RanBP9 under conditions of $10 \%$ fetal bovine serum (FBS), we detected only a marginal increase in lactate dehydrogenase (LDH) release (Figure 1a). However, when cells were stressed in $2 \%$ FBS, RanBP9-induced $\mathrm{LDH}$ release was marked accentuated (Figure 1a). To detect early and late stages of apoptosis, we used the Annexin-V/propidium iodide (PI) cell death assay under 10\% FBS conditions. The bottom left quadrant indicates healthy cells, whereas the bottom right and upper right quadrants indicate cells in early and late stages of apoptosis, respectively, in this assay (Figure 1b). Indeed, RanBP9 transfection reduced healthy cells and increased cells in early but not late stages of apoptosis in
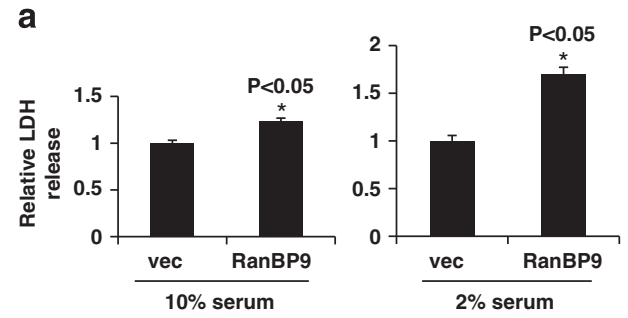

c
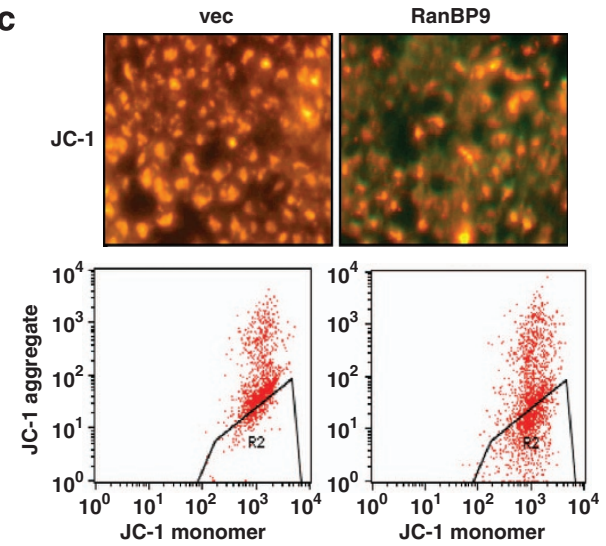

b
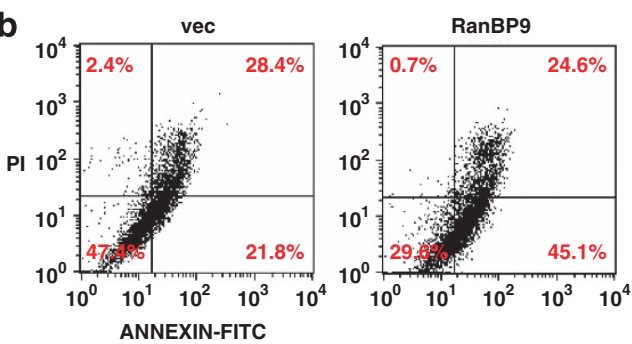

d
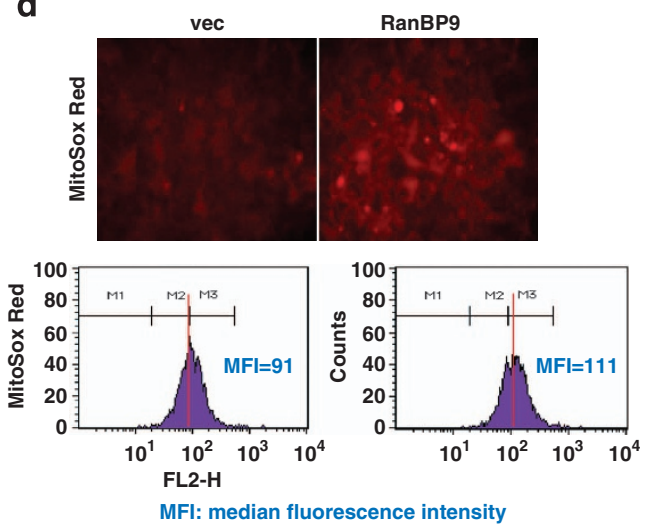

Figure 1 RanBP9 promotes cell death associated with increased mitochondrial superoxides and loss of MMP. (a) Graph shows LDH release in vector- or RanBP9transfected cells cultured with 10 and $2 \%$ fetal bovine serum (FBS) ( $n=3$ each). Error bars represent S.E.M. (b) Vector- or RanBP9-transfected HT22 cells were cultured in $10 \%$ FBS. After $48 \mathrm{~h}$, cells were subjected to Annexin V and PI staining followed by flow cytometry. A representative experiment from at least three independent experiments is shown. (c) HT22 cells were transfected with vector or RanBP9. After $36 \mathrm{~h}$, JC-1 staining was observed by fluorescence microscopy. Red and green fluorescence represent aggregated and monomeric JC-1. JC-1 staining was also observed by flow cytometry. Note that the R2 area shows the accumulation of monomeric JC-1 in RanBP9transfected cells. (d) MitoSOX Red staining was observed by fluorescence microscopy (upper) and flow cytometry (lower). Note the increase in the MitoSOX red signal in RanBP9 transfected cells. Representative experiments from at least three independent experiments are shown 
$10 \%$ FBS (Figure 1b). Therefore, RanBP9 appears not to induce overt cell death under normal culture conditions but increases the vulnerability of cells to undergo cell death under stressed conditions.

As mitochondrial oxidative stress and mitochondrial membrane potential (MMP) are general indicators of mitochondrial health, we next utilized the MitoSox Red, an indicator of mitochondrial superoxides, and JC-1, an indicator of MMP, to stain transiently transfected HT22 cells grown in 10\% FBS. Indeed, a consistent feature of the early stages of apoptosis is an increase in mitochondrial membrane permeability and loss of MMP. ${ }^{27}$ As the JC-1 dye exhibits potential-dependent accumulation in mitochondria and fluorescence shift from green $(529 \mathrm{~nm})$ to red $(590 \mathrm{~nm})$, mitochondrial depolarization is indicated by a decrease in red/green fluorescence intensity ratio. RanBP9 transfected HT22 cells displayed greatly decreased MMP as evidenced by the reduction and increase in red and green JC-1 fluorescence, respectively, compared with vector-transfected cells (Figure 1c, upper panels). Examination of red fluorescence by FACS analysis of JC-1 also demonstrated consistent findings, indicating that RanBP9 overexpression impairs MMP under conditions that do not exert overt cell death (Figure 1c, lower panels). MitoSox Red staining to measure mitochondrial superoxide levels also demonstrated much stronger red fluorescence in RanBP9 versus vector-transfected cells, indicating increased production of mitochondrial ROS (Figure 1d, upper panels). Further examination of MitoSox Red by FACS analysis also demonstrated similar results, with RanBP9-transfected cells displaying median fluorescence intensity of 111 versus 91 in vector-transfected cells (Figure 1d, lower panels). These results taken together indicate that RanBP9 increases the vulnerability of cells to undergo apoptosis and mitochondrial dysfunction even under conditions where overt cell death is not readily detectable.

Overexpression of RanBP9 alters $\mathrm{Bax} / \mathrm{Bcl} 2$ protein ratio, promotes Bax oligomerization, and induces cytochrome $\boldsymbol{c}$ release. It has been shown that knockdown of RanBP9 decreases mitochondrial $\mathrm{Bax}$ and increases $\mathrm{Bcl} 2$ in Hela cells. ${ }^{13}$ To determine whether corresponding changes are similarly seen after RanBP9 overexpression in brain-derived cells, we analyzed Bax and Bcl2 protein levels after control vector or RanBP9 transfection in HT22 cells. Indeed, Bcl2 levels were markedly decreased after RanBP9 transfection either under 10 or $2 \%$ FBS culture conditions, and Bax levels were moderately increased in $10 \%$ FBS and further increased in $2 \%$ FBS (Figure 2a). As transfection efficiency could dilute the effects of RanBP9 observed from total cell lysates, we also transfected EGFP or EGFP-RanBP9 and visualized Bax staining in EGFP-positive cells. Under these conditions, little to no Bax fluorescence was observed in EGFP-transfected cells (Figure 2b). However, EGFPRanBP9-transfected cells demonstrated significantly higher levels of Bax immunoreactivity, such that Bax immunofluorescence intensity in EGFP-RanBP9-transfected cells increased more than 2.5-fold compared with that of control EGFP-transfected cells in both 2 and 10\% FBS conditions (Figures $2 \mathrm{~b}$ and $\mathrm{c}$ ). Not all cells that were positive for EGFP. RanBP9 were positive for Bax, but most cells positive for
Bax were also positive for EGFP-RanBP9, suggesting cells in different stages of apoptosis induced by RanBP9 (Figure 2b). To determine whether RanBP9-induced increase in Bax protein might be due to increased Bax transcription, we next subjected vector- and RanBP9-transfected cells in 10 and $2 \%$ FBS to real-time quantitative reverse transcription-PCR (RT-qPCR). However, we observed no significant differences between vector and RanBP9-transfected cell in Bax mRNA levels, indicating that the increase in Bax protein is not due to a transcriptional mechanism (Figure 2d). To determine whether overexpression of RanBP9 induces Bax oligomerization, we applied anti-Bax antibody to detect monomer, dimer, and tetramer of the Bax protein. Both the 23-kD monomer and SDS-resistant 46-kD dimer were significantly increased in RanBP9-transfected cell compared with vector-transfected cells (Figure $2 \mathrm{e}$ ), indicating that RanBP9 promotes Bax oligomerization leading to increased mitochondrial membrane permeability.

Higher Bax/Bcl2 ratio, increased Bax oligomerication, and decreased MMP lead to the release of cytochrome $c$, a proapoptotic protein that promotes caspase activation and initiation of mitochondria-mediated apoptosis. To determine whether overexpression of RanBP9 induces the release of cytochrome $c$, we first subjected EGFP or EGFP-RanBP9transfected cells to immunofluorescence for cytochrome $c$ (red) in cells cultured in the normal 10\% FBS condition. Analysis of the double green/red fluorescent images showed that while EGFP-transfected cells largely contained cytochrome $c$ in discrete structures resembling mitochondria, EGFP-RanBP9-transfected cells also showed cytochrome $c$ diffusely in the cytoplasm (Figure 2f). Further biochemical isolation of mitochondria and cytosolic fractions also demonstrated similar findings. Specifically, RanBP9-transfected cells contained reduced and increased cytochrome $c$ in cytosolic and mitochondrial fractions, respectively, indicating its release from mitochondria to cytosol (Figure $2 \mathrm{~g}$ ). No cytosolic cytochrome $c$ was detected in control vectortransfected cells (Figure 2g). Surprisingly, RanBP9 was strongly detected in the isolated mitochondrial fraction together with the inner mitochondrial membrane marker Timm50 (Figure 2g), suggesting a potential direct role of RanBP9 in mitochondria. Taken together, these data indicate that RanBP9 induces mitochondrial dysfunction and renders cells more vulnerable to apoptosis by increasing mitochondrial ROS, decreasing MMP, increasing the $\mathrm{Bax} / \mathrm{Bcl}-2$ ratio, promoting Bax oligomerization, and releasing cytochrome $c$ release from mitochondria.

RanBP9-induced fragmentation of mitochondria and apoptosis is partially prevented by inhibition of mitochondrial fission. We next determined whether RanBP9 alters mitochondrial morphology under conditions of 10 and $2 \%$ FBS. HT22 cells were cotransfected with vector control or Flag-RanBP9 and Mito-dsRed to assess the shape and length of mitochondria. Compared with vector-transfected cells, RanBP9-transfected cells displayed marked fragmentation of mitochondria in both 10 and $2 \%$ FBS, although $2 \%$ FBS further increased mitochondrial fragmentation in both control- and RanBP9-transfected cells (Figure 3a). Quantitation of mitochondrial length indeed demonstrated more than 
a

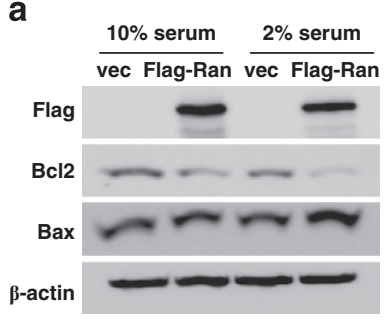

b
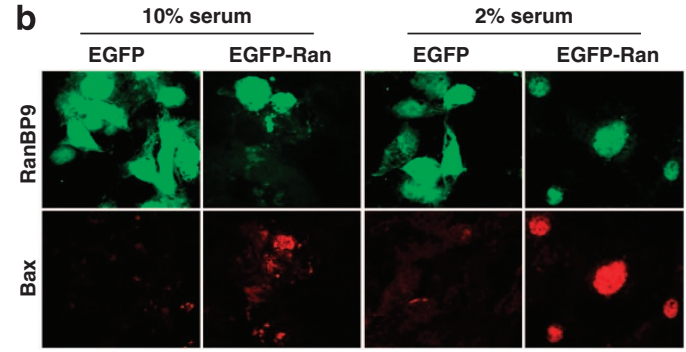

C

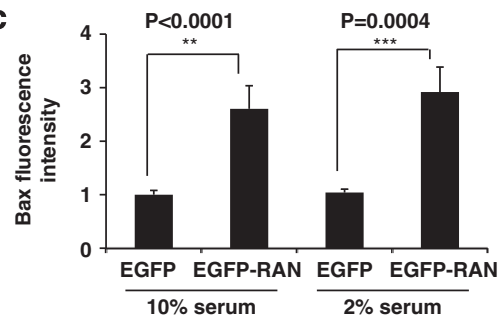

d

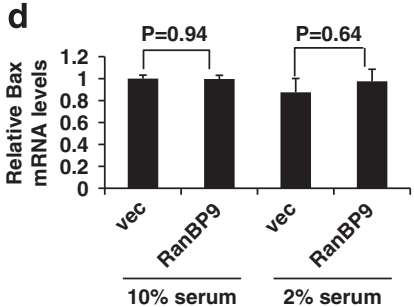

e

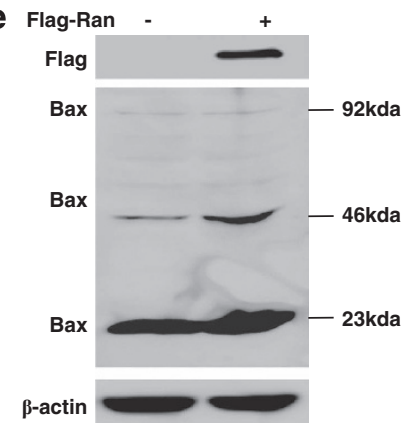

f

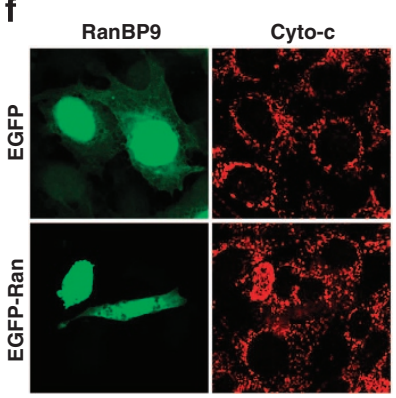

g

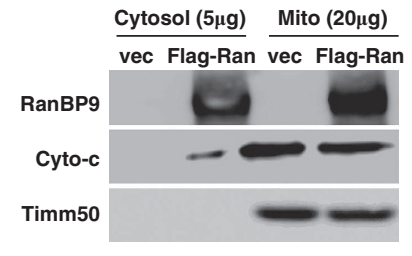

Figure 2 Increased Bax/Bcl-2 ratio and cytochrome $c$ release in RanBP9-transfected cells. HT22 cells were transfected with vector or RanBP9 for $24 \mathrm{~h}$, and cells were incubated in medium containing 10 or $2 \%$ FBS for another $24 \mathrm{~h}$. (a) Equal protein amounts of cell lysates were subjected to immunoblotting for Flag, Bax, Bcl-2. Note the relative decrease in Bcl-2 and increase in Bax levels in RanBP9-transfected cells. (b) Representative images of EGFP, EGFP-RanBP9, and Bax immunofluorescence. (c) Quantitation of Bax immunofluorescence intensity from EGFP or EGFP-RanBP9-transfected cells ( $n=4$ each). Error bars represent S.E.M. (d) Quantitative RT-PCR analysis of Bax mRNA levels normalized to GAPDH ( $n=4$ each). Error bars represent S.E.M. (e) HT22 cells were transfected with vector or RanBP9 for $48 \mathrm{~h}$, and equal protein amounts of cell lysates were subjected to immunoblotting for Bax. Note the increase in SDS-resistant 46-kD dimeric bax induced by RanBP 9 transfection. (f) Thirty-six hours after transfection of EGFP or EGFP-RanBP9, HT22 cells cultured in 10\% FBS were subjected to immunofluorescence for cytochrome $c$. A representative image shows widespread diffuse cytochrome $c$ staining in EGFP-RanBP9-transfected cells, suggestive of release to cytosol. (g) Thirty-six hours after transfection of EGFP or EGFP-RanBP9, HT22 cells cultured in 10\% FBS were subjected to biochemical isolation of mitochondria and cytosol. Representative experiment shows the localization of RanBP9 in both cytosol and mitochondria as well as release of cytochrome $c$ from mitochondria to cytosol in RanBP9-transfected cells. Timm50 was used as a marker of mitochondria

two-fold shorter mitochondria in RanBP9- versus controltransfected cells in both 10 and $2 \%$ FBS conditions (Figure $3 b$ ). The process of mitochondrial fission mediated by DRP1 not only participates in regulating mitochondrial morphology but is also important for the apoptotic process. ${ }^{28}$ It has been reported that DRP1 inhibitor mdivi-1 inhibits both mitochondrial fission and apoptosis. ${ }^{29}$ To determine whether RanBP9-induced mitochondrial fragmentation and apoptosis might be in part due to increased mitochondrial fission, we applied mdivi-1 to control and RanBP9-transfected cells in $2 \%$ FBS and monitored both mitochondrial fragmentation and cell death. Indeed, mdivi-1 significantly prevented mitochondrial fragmentation (Figure $3 \mathrm{c}$ ) and apoptosis (Figure 3d) induced by RanBP9, indicating that the process of mitochondrial fission is an integral component of RanBP9induced cell death.
Anti-apoptotic proteins XIAP, Bcl-2 and Bcl-xl suppress RanBP9-induced toxicity. X-linked inhibitor of apoptosis protein (XIAP) inhibits apoptotic cell death by inhibiting caspases 3,7 and 9, the latter that is activated by cytochrome $c$ release from mitochondria. ${ }^{30}$ Both $\mathrm{Bcl}-2$ and $\mathrm{Bcl}-\mathrm{xl}$ are mitochondrial proteins that suppress apoptosis by inhibiting mitochondrial membrane permeability and cytochrome $c$ release. ${ }^{31}$ As RanBP9 promoted the release of cytochrome $c$ and induced mitochondrial dysfunction, we next assessed whether XIAP, Bcl-2 and Bcl-xl can inhibit RanBP9-induced cell death. Therefore, HT22 cells were cotransfected with vector or RanBP9 together with XIAP, Bcl-2 or Bcl-xl for $48 \mathrm{~h}$ and subjected to $2 \%$ FBS treatment for $24 \mathrm{~h}$. Under these conditions, RanBP9 increased both early (lower right quadrant) and late apoptotic (upper right quadrant) cells as assessed by Annexin V/PI staining followed by FACS 
a
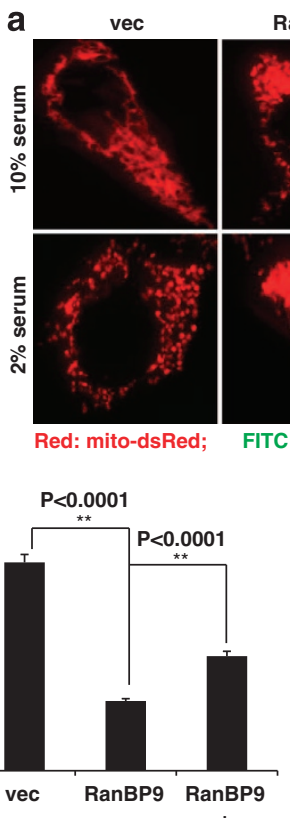

RanBP9

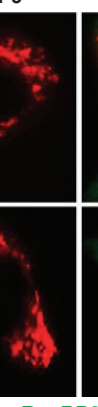

Merge

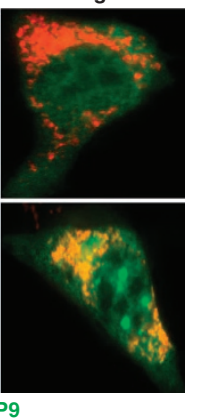

b

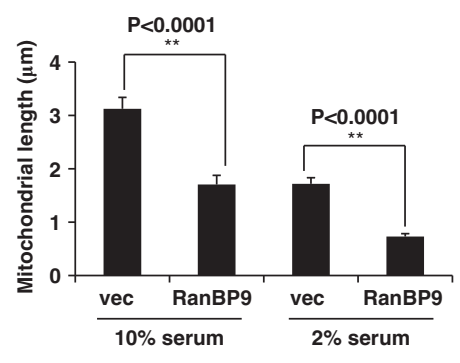

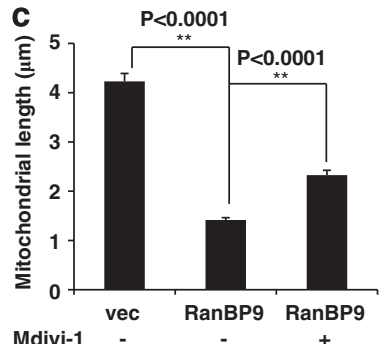

d
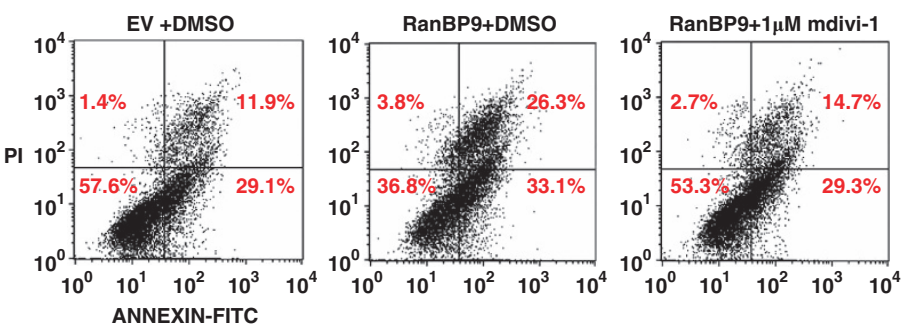

Figure 3 Inhibition of RanBP9-induced mitochondrial fission reduces cell death. $(\mathbf{a}, \mathbf{b})$ HT22 cells were transfected with vector or RanBP9 together with mito-dsRed for $24 \mathrm{~h}$, and cells were incubated in medium containing 10 or $2 \%$ FBS for another $24 \mathrm{~h}$. Cells were fixed with $4 \%$ PFA and subjected to immunofluorescence for Flag-RanBPg (M2; green) visualization of mito-dsRed signal (red). Representative images show increased fragment of mitochondria (mito-dsRed) in RanBP9-transfected cells, which is further fragmented in $2 \%$ FBS. (b) Quantitation of mitochondrial length in RanBP9 or vector-transfected cells $(n=4$ each). Error bars represent S.E.M. At least 10 cells were selected in each sample and about 50 mitochondria were measured from each selected cell. (c, d) HT22 cells cotransfected with vector or RanBP9 together with mito-dsRed were treated with or without the mitochondrial fission inhibitor mdivi-1 $(5 \mu \mathrm{M})$ for $12 \mathrm{~h}$, and mitochondrial length was measured using the NIS-Elements-AR software $(n=3$ each). Error bars represent S.E.M. At least 10 cells were selected in each sample and about 50 mitochondria were measured from each selected cell. Alternatively, cells were subjected to Annexin V/PI staining followed by FACS analysis. A representative cell death experiment is shown from at least three independent experiments

analysis (Figures 4a and b). However, XIAP, Bcl-2 and Bcl-xl were all highly effective in suppressing RanBP9-induced apoptosis (Figures $4 \mathrm{a}$ and $\mathrm{b}$ ), further supporting the notion that RanBP9 induces cell death via a mitochondria- and caspase-dependent apoptotic pathway.

RanBP9 enhances transcriptionally active p73 levels by both transcriptional and post-translational mechanisms. It has been reported that RanBP9 physically associates with $\mathrm{p} 73 \alpha$ and increases $\mathrm{p} 73 \alpha$ levels, ${ }^{25}$ though the precise mechanism of such action is unclear. p73, much like its cousin p53, functions as a tumor-suppressor protein and induces apoptosis via both transcriptional and mitochondrial pathways. ${ }^{26}$ The p73 protein and its cleaved fragments have been detected in mitochondria during apoptosis induced by tumor necrosis factor-related apoptosis-inducing ligand (TRAIL). ${ }^{32}$ Consistent with previous observations, we detected the physical association between RanBP9 and $\mathrm{p} 73 \alpha$ in coimmunoprecipitation experiments (Figure 5a). Indeed, RanBP9 overexpression increased the total amount of endogenous p73 protein (Figure $5 b$ ) and also enhanced the level of cotransfected $\mathrm{p} 73 \alpha$ in biochemically isolated mitochondria (Figure 5c). RanBP9 was again detected in isolated mitochondrial fractions (Figures $5 b$ and $c$ ), and the N-terminal $60-\mathrm{kD}$ fragment (N60) of RanBP9 that is increased in $A D$ brains, was preferentially localized to mitochondria (not shown). To determine whether RanBP9 stabilizes endogenous p73, HT22 cells were cotransfected with vector control or RanBP9, and cells were subjected to cycloheximide $(\mathrm{CHX})$ chase assay over $4 \mathrm{~h}$. Indeed, RanBP9 markedly increased the half-life of endogenous $p 73$, indicating the stabilization of $p 73$ at the post-translational level. As previous studies have shown that RanBP9 can also act in the nucleus as a transcriptional co-regulator, ${ }^{33-35}$ we also determined whether RanBP9 alters the level of endogenous p73 mRNA. Quantitative real-time reverse transcription (RT) PCR analysis showed that RanBP9 increased the level of p73 mRNA by $\sim 1.7$-fold in HT22 cells (Figure $5 e$ ), indicating that RanBP9 controls p73 levels and its mitochondrial content, both transcriptionally and post-translationally. To determine whether RanBP9 increases transcriptionally active p73, we also assessed the levels of p21 and puma mRNAs, the former activated by $\mathrm{p} 53$ and the latter activated by both p53 and p73. Quantitative real-time RT-PCR analysis demonstrated that RanBP9 overexpression significantly increased puma but not p21 mRNA levels, consistent with a specific increase in transcriptionally active p73 but not p53 (Figures 5e and f).

Endogenous RanBP9 and p73 cooperate to induce mitochondrial dysfunction and apoptosis. Induction of p73 is expected to induce mitochondrial dysfunction and apoptosis. To determine whether p73 is essential for RanBP9-induced toxicity, HT22 cells were cotransfected with vector control or RanBP9 together with control siRNA (si NC) or pan p73 siRNA (pan-si p73) in 10\% FBS. As expected, RanBP9 
a

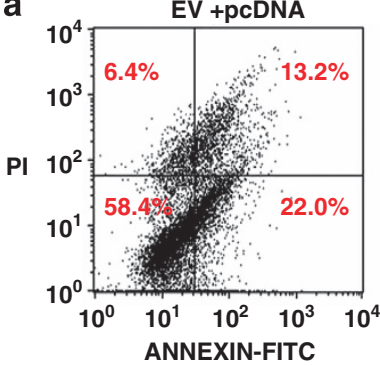

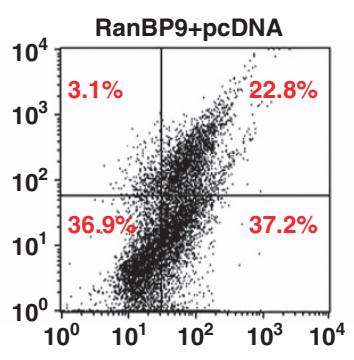

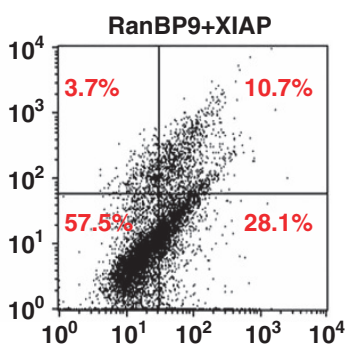

b
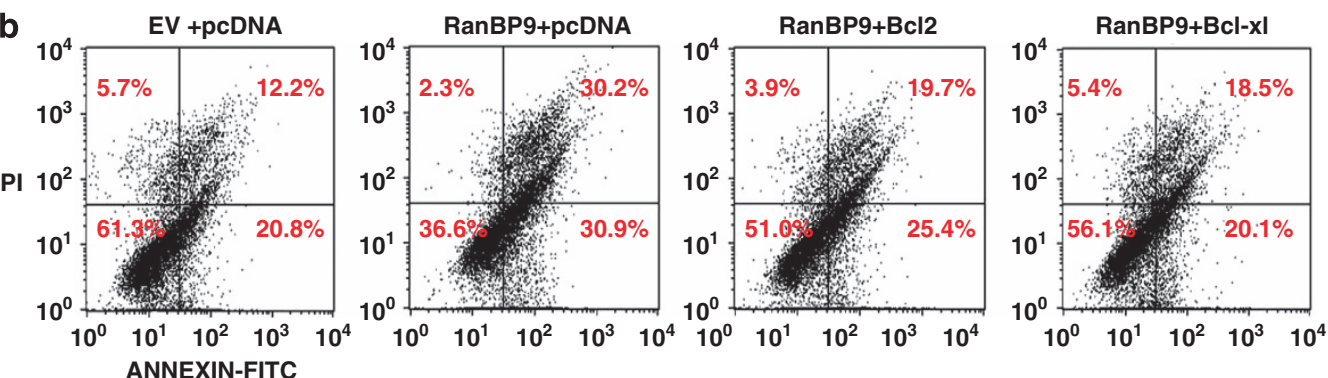

Figure 4 XIAP, Bcl-2 and Bcl-xl antagonize RanBP9-induced cell death. (a, b) HT22 cells were transfected with empty vector (EV) or RanBP9 or RanBP9 together with vector, XIAP, Bcl-2, or Bcl-xl. After $24 \mathrm{~h}$, the medium was replaced with new medium containing $2 \% \mathrm{FBS}$. After $24 \mathrm{~h}$, the cells were stained by Annexin $\mathrm{V}$ and PI, and apoptosis was measured using flow cytometry. Each independent experiment was reproduced at least three times, and representative experiments are shown
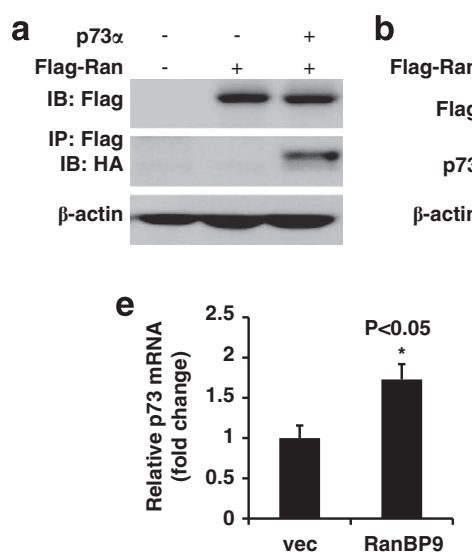

b

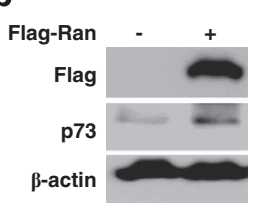

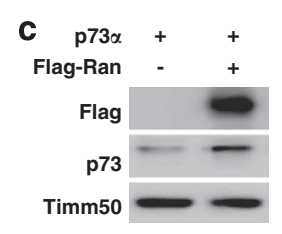

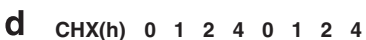
Flag-Ran - - - + + + +

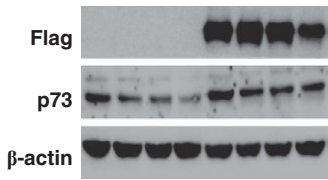

f

$$
\text { f }
$$
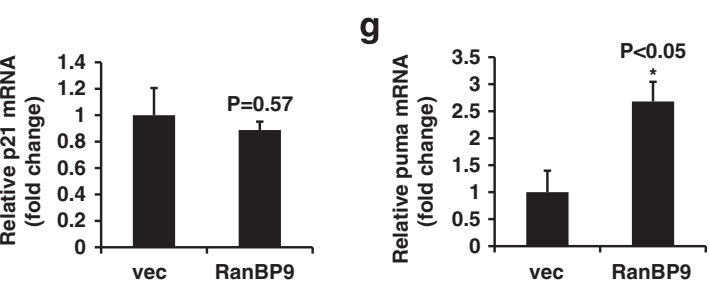

Figure 5 RanBP9 physically interacts with p73 and increases $p 73$ levels by both protein stabilization and increased transcription. (a) Flag-RanBP9 was cotransfected with or without HA-p73 in HT22 cells, and equal protein amounts of cell lysates were immunoprecipitated with anti-FLAG M2 antibody followed by immunoblotting for anti-HA antibody to detect HA-p73. (b) Increase in endogenous p73 after Flag-RanBP9 transfection in HT22 cells. Flag-RanBP9 and endogenous p73 were detected using M2 and anti-p73 antibodies, respectively. A representative experiment is shown. (c) p73 $\alpha$ was cotransfected with vector or RanBP9 in HT22 cells. After $48 \mathrm{~h}$, mitochondria were isolated, and equal protein amounts of lysates were immunoblotted using anti-FLAG M2, anti-p73 and anti-timm50 antibodies. (d) Vector and RanBP9 were transfected in HT22 cells. After $48 \mathrm{~h}$, cells were treated with $2 \mu \mathrm{g} / \mathrm{ml}$ cycloheximide (CHX) for $0,1,2$ and $4 \mathrm{~h}$. Equal protein amounts of cell lysate were subjected to immunoblotting using antiFLAG M2, anti-p73 and anti- $\beta$-actin antibodies. (e, $\mathbf{f}, \mathbf{g}$ ) Vector or RanBP9 were transfected in HT22 cells. After $48 \mathrm{~h}$, total RNA was isolated and subjected to quantitative RT-PCR using specific primers for TAp73, p21, and puma, and normalized to GAPDH ( $n=4$ each). Error bars represent S.E.M.

increased endogenous p73 and Bax and decreased Bcl-2 levels without altering $\beta$-actin (Figure 6a). However, cotransfection of RanBP9 with p73 siRNA completely reversed these effects of RanBP9 (Figure 6a). Likewise, p73 siRNA restored MMP (Figure 6b, lower panels), suppressed apoptosis (Figure 6b, upper panels), and reversed mitochondrial fragmentation (Figures $6 \mathrm{c}$ and d) induced by RanBP9. To determine whether RanBP9 might also be an essential component of p73 $\alpha$-induced cell death, HT22 cells were cotransfected with vector control or $\mathrm{p} 73 \alpha$ with or without RanBP9 siRNA and subjected to $2 \%$ FBS treatment for $24 \mathrm{~h}$. As expected, p73a transfection increased Bax, decreased $\mathrm{Bcl} 2$, and increased both early and late stages of apoptosis (Figures $6 e$ and f). Surprisingly, knockdown of RanBP9 also largely reversed the increase in Bax, decrease in $\mathrm{Bcl}-2$, and increase in cell death induced by $p 73 \alpha$ (Figures $6 e$ and f), suggesting that RanBP9 and $p 73 \alpha$ function cooperatively to induce mitochondrial dysfunction and cell death. 
a
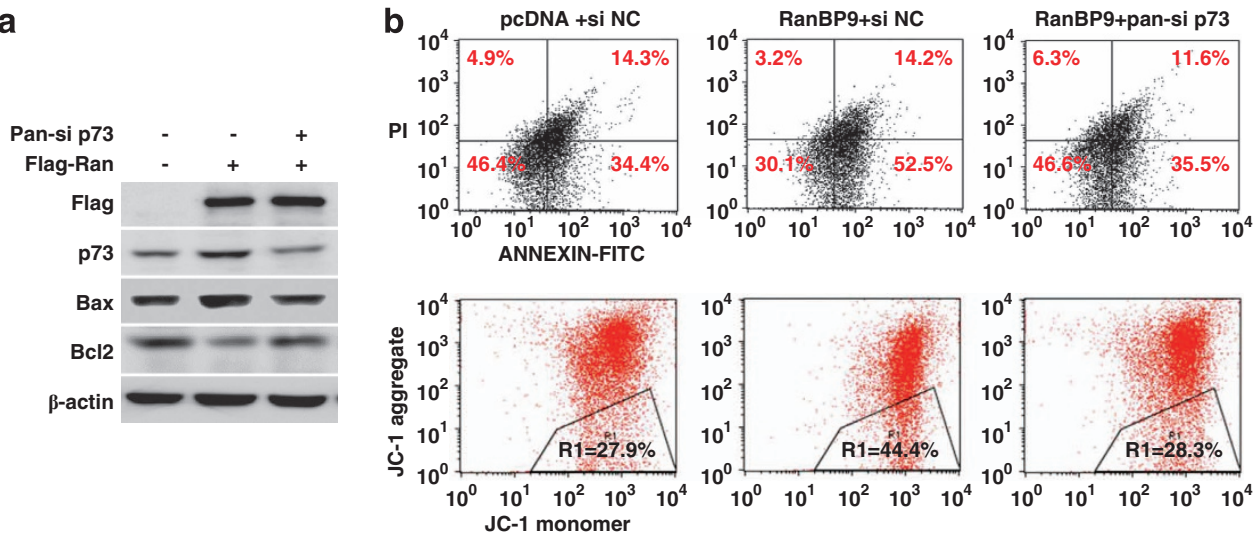

C

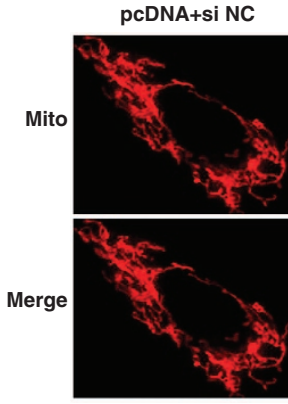

Red: mito-dsRed;
RanBP9+si NC

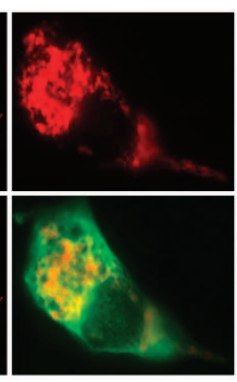

FITC: RanBP9

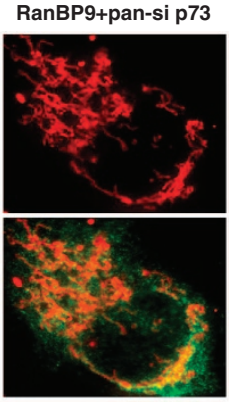

d

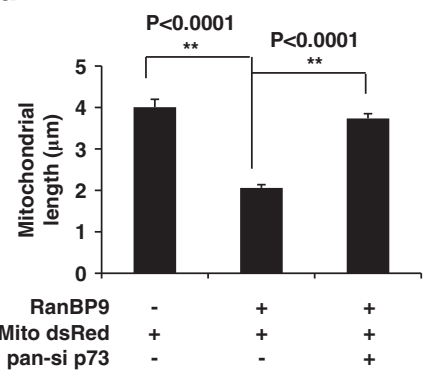

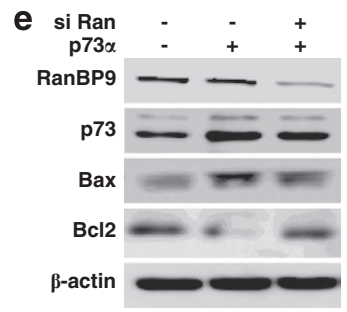

f
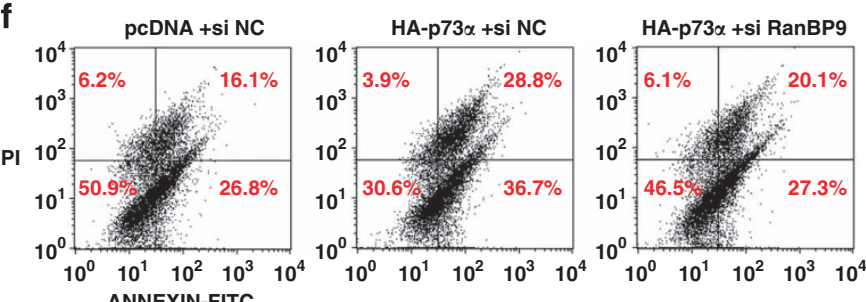

Figure 6 p73 and RanBP9 function cooperatively to induce mitochondrial dysfunction and cell death. RanBP9 was cotransfected with negative control siRNA (siNC) or pan-p73 siRNA (pan-si p73) for $48 \mathrm{~h}$ in HT22 cells. (a) Equal protein amounts of cell lysates were subjected to immunoblotting for Flag-RanBP9 (M2), p73, Bax, Bcl-2, and $\beta$ actin. (b) Cells were stained with Annexin V and PI or JC-1 and subjected to FACS analysis for cell death and MMP. (c, d) RanBP9 and Mito-dsRed were cotransfected with control siRNA (si NC) or pan-p73 siRNA (pan-si p73) in HT22 cells. After $72 \mathrm{~h}$, cells were fixed with 4\% PFA and subjected to immunofluorescence for Flag-RanBP9 (green) and visualization of mito-dsRed (red). Representative images are shown. Mitochondrial length was measured using Nikon NIS Elements-AR software from at least 10 cells per sample and about 50 mitochondria per selected cell. Error bars represent S.E.M. ( $n=4$ samples each). (e, f) HA-p73 $\alpha$ was cotransfected with control siRNA (si NC) or RanBP9 siRNA (si RanBP9 for $72 \mathrm{~h}$ in HT22 cells, and equal protein amounts of cell lysates were immunoblotted for endogenous RanBP9, p73, Bax, Bcl-2, and $\beta$-actin. Cells were also stained with Annexin V-FITC/PI and subjected to FACS analysis for cell death. A representative experiment is shown from at least three independent experiments

Endogenous $\mathrm{p73}$ is essential for $\mathrm{A} \beta$ - and RanBP9induced apoptosis and mitochondrial dysfunction in primary hippocampal neurons. To determine whether RanBP9 functions in a similar p73-dependent manner in inducing cell death in neurons, primary hippocampal neurons isolated from PO pups of RanBP9 transgenic (TG) and wildtype (WT) littermates were cultured in DIV14. Immunofluorescence analysis of endogenous p73 demonstrated a $\sim 2.5$-fold increase in p73 fluorescence intensity in RanBP9 transgenic neurons compared with nontransgenic wild-type neurons (Figures $7 \mathrm{a}$ and b). M2 staining for Flag-RanBP9 indeed demonstrated expression of the RanBP9 transgene in primary hippocampal neurons (Figure 7a). We also carried out immunofluorescence staining for cytochrome $c$. Prior to fixation, neurons were treated with $0.1 \%$ saponin to release cytosolic content and were then subjected to cytochrome $c$ immunofluorescence staining. Consistent with observations in HT22 cells, RanBP9 TG neurons demonstrated significantly reduced cytochrome $c$ fluorescence intensity (Figures $7 c$ and $d$ ), indicative of its release from mitochondria. Moreover, RanBP9 TG neurons also showed significantly reduced red JC-1 aggregates and increased green JC-1 monomers (Figures $7 e$ and f), indicative of reduced MMP.

To induce apoptotic cell death, DIV7 primary neurons were treated with $1 \mu \mathrm{M} \mathrm{A} \beta 1-42$ for $24 \mathrm{~h}$ with or without control siRNA (siNC) or pan-p73 siRNA (siRNAp73) transfection, and cell were then subjected to Annexin V (red) and DAPI staining (blue). Under these conditions, RanBP9 TG neurons demonstrated significantly increased percentage of Annexin V-positive apoptotic cells compared with WT controls (Figures $7 \mathrm{~g}$ 
a

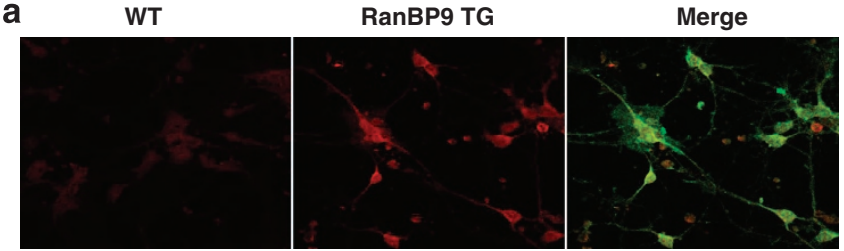

Red: p73;

FITC: Flag-RanBP9
C

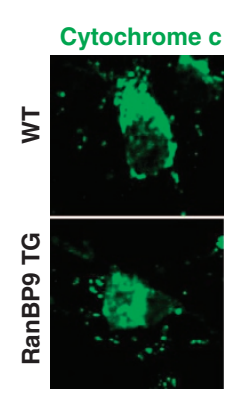

d

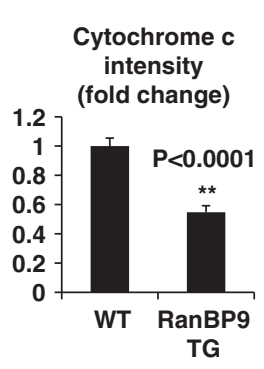

e Red: JC-1 aggregate Green: JC-1 monomer
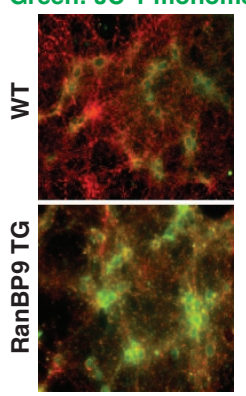

g WT+siNC

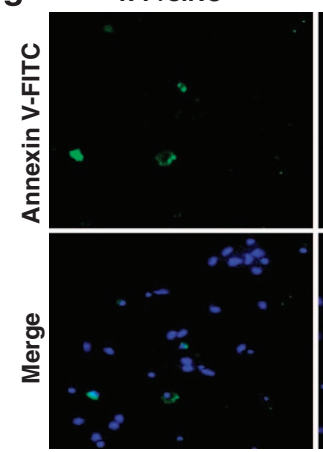

Annexin V-FITC; DAPI
RanBP9 TG+siNC
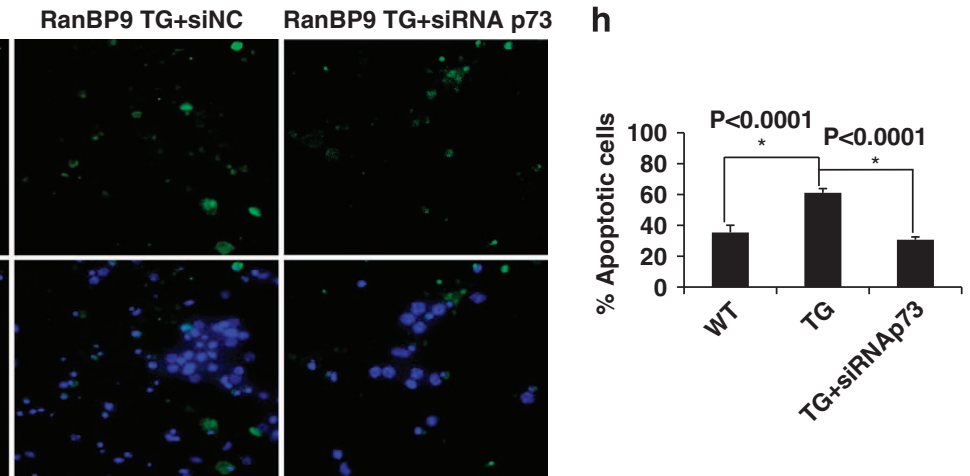

Figure 7 p73 is essential for RanBP9/A 1 1-42-induced apoptosis and mitochondrial dysfunction in primary hippocampal neurons. (a, b) RanBP9 transgenic (TG) and littermate non-transgenic (WT) DIV14 primary hippocampal neurons were subjected to immunostaining using anti-FLAG M2 and anti-p73 antibodies, and images were captured by confocal microscopy. Representative images are shown. Endogenous p73 fluorescence intensity (red) was quantified using Nikon NIS-Elements-AR software ( $n=4$ each). Error bars represent S.E.M. (c,d) RanBP9 transgenic (TG) and littermate non-transgenic (WT) DIV14 primary hippocampal neurons were treated with $0.1 \%$ saponin ( $5 \mathrm{~min}$ on ice) to release cytosolic content prior to fixation and subjected to immunostaining using anti-cytochrome $c$, and images were captured by confocal microscopy. Representative images are shown. (d) Endogenous cytochrome $c$ (FITC) intensities were quantified using Nikon NIS-Elements-AR software ( $n=4$ each). Error bars represent S.E.M. (e,f) Wild-type and RanBP9 transgenic littermate hippocampal primary neurons were subjected to JC-1 staining prior to fixation, and JC-1 signals were captured by fluorescence microscopy. (f) Red (JC-1 aggregate) and green (JC-1 monomer) fluorescence intensities from were quantified using NIS-Elements AR 3.2 software and relative quantifications are shown in the graph ( $n=4$ each). Error bars represent S.E.M. (g,h) Wild-type and RanBP9 transgenic littermate hippocampal primary neurons were transfected with control or p73 siRNA. After $48 \mathrm{~h}$, cells were treated with $1 \mu \mathrm{M} \mathrm{A} \beta$ for $24 \mathrm{~h}$ and subjected to staining with Annexin V-FITC and DAPI. Cells were captured directly using fluorescence microscopy. Numbers of Annexin V-FITC-positive cells among DAPI-positive cells were quantified using Nikon NIS-Elements-AR software. The graph shows \% Annexin V-positive apoptotic cells ( $n=3$ each). Error bars represent S.E.M.

and h). Consistent with observations in HT22 cells, p73 siRNA significantly prevented the increase in apoptosis induced by RanBP9 and A $\beta 1-42$ (Figures $7 g$ and $h$ ). Taken together, these observations demonstrate that RanBP9 and $p 73 \alpha$ function cooperatively likely in a protein complex to mediate mitochondria-mediated apoptosis in multiple cell types, including neurons.

\section{Discussion}

Mitochondrial dysfunction is widely believed to be an early and critical component of multiple neurodegenerative diseases, including $\mathrm{AD}$. Accumulations of $\mathrm{A} \beta$ and $\tau$ are both associated with mitochondrial dysfunction at multiple levels. ${ }^{1-3} \mathrm{We}$ previously showed that the overall levels of RanBP9 are elevated in brains of AD patients and in APP transgenic mice and that increased RanBP9 expression promotes $\mathrm{A} \beta$ generation both in cultured cells and in brain (Figure 8). ${ }^{19-21}$ Furthermore, we demonstrated that RanBP9 induces neurodegeneration and synaptic loss associated with spatial memory deficits and that RanBP9 is essential to mediate $\mathrm{A} \beta$-induced neurotoxicity. ${ }^{23}$ In this study, we made a series of novel observations implicating the cooperative role of the RanBP9/p73 complex in mitochondrial dysfunction and 


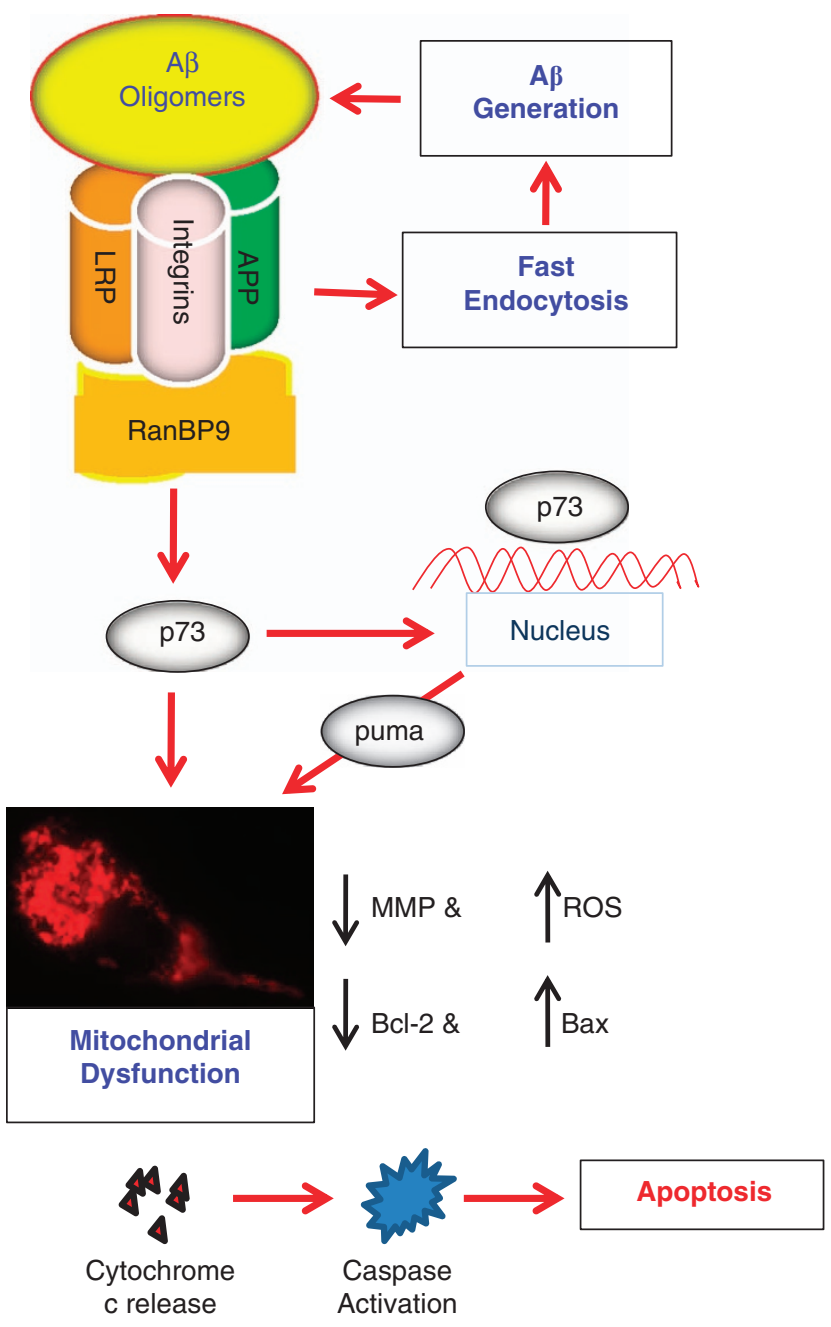

Figure 8 Schematic model of the RanBP9/p73 pathway in neurodegeneration and $A D$ pathogenesis. As RanBP9 levels are elevated in brains of AD patients, the binding of RanBP9 to integrin/APP/LRP complexes accelerates their endocytosis, thereby simultaneously promoting $A \beta$ generation and disrupting focal adhesions. $\mathrm{A} \beta$ oligomers via binding to integrin complexes further propagate their toxic signals. Meanwhile, $\mathrm{A} \beta$ and RanBP9 function to stabilize $\mathrm{p} 73 \alpha$ and/or activate its transcription. p73 translocates into the nucleus and mitochondria to induce puma, increase Bax, reduce Bcl-2, drop MMP, and enhance mitochondrial ROS, leading to increased mitochondrial permeability, release of cytochrome $c$, and apoptosis

apoptosis. First, we showed that RanBP9 induces the loss of MMP and increased mitochondrial superoxide levels even under conditions in which overt cell death was not readily detected. Such changes were associated with increased Bax level and oligomerization, decreased $\mathrm{Bcl}-2$, and release of cytochrome $c$ from mitochondria. Second, overexpression of RanBP9 led to the fragmentation of mitochondria and cell death, which were significantly reversed by the mitochondrial fission inhibitor Mdivi-1. Third, RanBP9-induced apoptosis was efficiently blocked by classical inhibitors of mitochondrial cell death pathway, XIAP, Bcl-2, and Bcl-xl. Fourth, RanBP9 increased endogenous p73 levels and promoted p73 localization in mitochondria at both transcriptional and post-translational levels. Fifth, RanBP9 increased transcriptionally active p73 as evidenced by increased transcription of the p73 target gene, puma. Sixth, knockdown of endogenous p73 by siRNA effectively blocked RanBP9-induced changes in cell death, MMP, and mitochondrial fragmentation, indicating that p73 is an essential component of RanBP9-induced apoptosis. Seventh, RanBP9 increased p73 levels and cytochrome $c$ release primary hippocampal neurons, and siRNA knockdown of p73 also antagonized RanBP9/A $\beta 1-42$ induced apoptosis in primary neurons. Finally, siRNA knockdown of endogenous RanBP9 was also effective in suppressing p73-induced apoptosis, suggesting that RanBP9 and p73 have cooperative roles in inducing cell death. Taken together, these finding implicate the RanBP9/p73 complex in mitochondrial dysfunction and apoptotic mechanisms during neurodegeneration.

Previous studies have shown that RanBP9 can exert proapoptotic activity during DNA damage-induced cell death and that RanBP9 physically associates with $p 73 \alpha$ and stabilizes exogenously expressed $p 73 \alpha$ protein. ${ }^{13,25}$ In this study, we found that RanBP9 increases endogenous $p 73$ by protein stabilization as well as increased transcription of p73 and its target gene, puma, and that endogenous p73 is essential for RanBP9-induced apoptosis, mitochondrial fragmentation, and loss of MMP (Figure 8). Furthermore, RanBP9 itself and p73 were increased in mitochondria secondary to RanBP9 overexpression. We also recently reported that RanBP9 is critical for A $\beta 1$-42-induced cell death and that RanBP9 activates cofilin, ${ }^{23}$ an actin-binding protein that regulates actin dynamics and mitochondrial function. ${ }^{36-38}$ Indeed, knockdown of cofilin was able to prevent both RanBP9- and A $\beta$-induced apoptosis. ${ }^{23}$ Previous studies have shown that recombinant $\mathrm{p} 73$ or recombinant cofilin alone is capable of inducing the release of cytochrome $c$ from isolated mitochondria due to increased permeability, ${ }^{32,39}$ indicating direct effects on mitochondria. Such findings are entirely consistent with our observations that RanBP9 overexpression increased mitochondrial permeability as seen by increased bax oligomerization, decreased MMP, associated with a rise in mitochondrial superoxides. Therefore, RanBP9 appears to promote apoptosis via both cofilin and p73-dependent mechanisms, possibly with direct effects on mitochondria. Although it has been reported that RanBP9 potentiates the transcriptional activation of the p73-responsive gene, MDM2 ${ }^{25}$ we did not detect changes in Bax mRNA levels despite the increase in Bax protein. Nevertheless, we confirmed that RanBP9 induces the transcription of another p73-responsive gene, puma, but not the p53-responsive gene, p21. It has been demonstrated that even transcriptionally inactive p73 is capable of inducing apoptosis via a mitochondrial mechanism. ${ }^{32}$ Therefore, it is likely that RanBP9-induced changes in mitochondrial function and apoptosis can be attributed to both transcriptional (i.e. puma) and non-transcriptional mechanisms, the latter bearing directly on mitochondria. Consistent with a previous study demonstrating the physical association between RanBP9 and p $73 \alpha,{ }^{25}$ we confirmed this interaction by coimmunoprecipitation studies. Interestingly, we found that knockdown of RanBP9 by siRNA also largely eliminated the toxic effects of p73 $\alpha$, suggesting that p73 and RanBP9 function cooperatively in a protein complex to mediate mitochondrial dysfunction and cytotoxicity. Whether cofilin might be a component of that protein complex remains to be determined. 
We found that RanBP9 increased endogenous $p 73 \alpha$ levels, increased cytochrome $c$ release, and promoted apoptosis induced by $A \beta 1-42$ treatment in primary hippocampal neurons, which was reversed by p73 pan-siRNA. Although it has been reported that the $\mathrm{N}$-terminally truncated p73 $(\Delta \mathrm{Np} 73)$, which promotes cell survival, is a major species expressed in neurons, ${ }^{40,41}$ the antibody we used to detect p73 does not detect $\triangle N p 73$ as it is directed against the $\mathrm{N}$-terminal 80 residues of $\mathrm{p} 73$. Furthermore, the siRNA that we had used to knockdown p73 targets all forms of p73. Hence, in primary hippocampal neurons used in our system, toxic forms of p73 are induced by RanBP9, which when suppressed by siRNA, render neurons protected against RanBP9/A 11-42-induced apoptosis. Indeed, it has been shown that the toxic p73 species are widely expressed in adult human brains. ${ }^{42,43}$ As RanBP9 levels are increased in AD brains and in brains of APP transgenic mice, ${ }^{21,23}$ it is expected that the RanBP9/p73 complex would promote mitochondrial dysfunction and render neurons more vulnerable to degeneration. Consistent with this notion, it has been reported that nuclear p73 accumulates in brains of $A D$ patients, ${ }^{43}$ and $p 73 \alpha$ promotes the phosphorylation of $\tau$ via a transcription-dependent mechanism. ${ }^{44}$ Although the loss of $\Delta \mathrm{Np} 73$ is associated with neurodegeneration and $\tau$ hyperphosphorylation, ${ }^{40,45}$ it has been shown that $A \beta$ increases p73 $\alpha$ immunoreactivity in vivo as well as in cultured primary neurons and reduces the neuroprotective $\Delta N p 73$ isoform. ${ }^{46,47}$ In view of these observations together with the results of the current study, we hypothesize that targeting the p $73 \alpha /$ RanBP9 complex and downstream pathway may be promising therapeutic strategy to simultaneously antagonize neurodegeneration and $\mathrm{A} \beta$ generation.

\section{Materials and Methods}

Cell culture. Wide-type and RanBP9 transgenic mice were generated as previously reported. ${ }^{20,23}$ Mouse hippocampal primary neurons from $\mathrm{P} 0$ pups were cultured in Neurobasal medium supplemented with $1 \times$ B-27 supplement and $1 \times$ L-Glutamine (Invitrogen, CA, USA) in a humidified atmosphere of $5 \% \mathrm{CO} 2$ at $37^{\circ} \mathrm{C}$. The medium was replaced every 2-3 days. Cells were cultured in Dulbecco's modified Eagle's medium (DMEM, Thermo Scientific, MA, USA) supplemented with $10 \%$ fetal bovine serum) and $1 \%$ penicillin/streptomycin $(\mathrm{P} / \mathrm{S})$ in a humidified atmosphere of $5 \% \mathrm{CO} 2$ at $37^{\circ} \mathrm{C}$.

Cell death assays. HT22 cells were cultured in DMEM medium with $1 \%$ penicillin/streptomycin and 10\% FBS in a 24-well plate. After 24-h incubation, transfection was performed using lipofectamine 2000 according to the manufacturer's instructions. Forty-eight hours after transfection and in the presence of $10 \%$ or $2 \%$ FBS for $24 \mathrm{~h}, 200 \mu \mathrm{l}$ of culture medium were collected and used in LDH release assays. LDH level was measured with In vitro Toxicology assay kit (Sigma-Aldrich, MO, USA) by spectrophotometer following the manufacturer's instructions. For annexin V/PI cell death assays, the Annexin-VFITC and PI Apoptosis Detection Kit (BD Bisosciences) was used, followed by FACS analysis (FACS Calibur, BD, CA, USA) according to the manufacturer's instructions and as previously documented. ${ }^{23}$ For JC-1 staining and MitoSox Red staining, after the cells were harvested and washed with PBS, they were stained with JC-1(Invitrogen) or MitoSox (Invitrogen) Red for $15 \mathrm{~min}$. Cells were washed once with binding buffer and measured by flow cytometry or captured by fluorescence microscopy. In primary hippocampal neurons, JC-1 staining was done for $15 \mathrm{~min}$ prior to fixation and detected directly on plates by fluorescence microscopy. Quantitations were performed by selecting the cellular region of 20-30 cells for each sample and measuring the intensity of green and red signals using Nikon NIS Elements-AR software. For Annexin V cell death assay, DIV7 neurons were treated with $A \beta 1-42$ for $24 \mathrm{~h}$ and stained with Annexin V-FITC followed by DAPI (BD, San Diego, CA, USA).
Immunoblotting and mitochondrial isolation. Cell lysate was lysed with lysis buffer (50 mM Tris-Cl,150 mM NaCl, 2 mM EDTA and 1\% Triton-100) and total protein concentration was quantified by a colorimetric detection assay (BCA Protein Assay, Pierce, USA). Equal amounts of protein lysates were separated by sodium dodecyl sulfate-polyacrylamide gel electrophoresis, and transferred to Immobilon-P membranes (Millipore Corporation, Bedford, MA, USA). Interested proteins were probed by primary antibodies and corresponding peroxidase-labeled secondary antibodies, followed with detection by ECL (Millipore Corporation). Mitochondria were isolated by Mitochondrial Isolation Kit for Cultured Cells (Thermo Scientific, IL, USA) according to the manufacturer's instructions.

Transient transfections. Transient transfections of HT22 cells and 293T cells with DNA plasmid were carried out using lipofectamine 2000 (Invitrogen, Carlsbad, CA, USA) and Opti-MEM I (Invitrogen). For siRNA transfections, lipofectamine RNAiMAX (Invitrogen) and Opti-MEM I were applied. Four to six hours following transfections, the medium was replaced with new complete medium. Generally, cells were incubated $48 \mathrm{~h}$ after plasmid transfection and $72 \mathrm{~h}$ after siRNA transfection.

DNA constructs and siRNA. pEGFP-C2-RanBP9 was a kind gift from Dr. Hideo Nishitani (Kyushu University, Fukuoka, Japan). pcDNA-P3X-FlagRanBP9 construct was a gift from Dr. Shim S-K (Yale University School of Medicine, New Haven, CT, USA). pLHCX RanBP9 and RanBP9-N60 constructs have previously been described. ${ }^{19}$ pCMV-XIAP, pcDNA-myc-Bcl2 and pcDNAmyc-Bcl-xl constructs were gifts from Dr. Ryu Hoon (Boston University, Boston, USA). RanBP9 siRNA was synthesized by Samchully Pharm (Seoul, Korea) with the following sequence: sense UCUUAUCAAUACCUGCTT; antisense GCUGGUAUUGUUGAUAAGATT. Pan-p73 siRNA was synthesized by Biobeer (Korea) with the following sequence: sense $5^{\prime}$-CCAUCCUGUACAACUUCAUGU G-3', antisense 5'-CAUGAAGUUGUACAGGAUGGU G-3. pcDNA-HA-p73 $\alpha$ and $\triangle N p 73 \alpha$ constructs were generously provided by Dr. Nakagawara (Japan).

Chemicals and antibodies. RanBP9 monoclonal antibody was a generous gift from Dr. Bianchi (Pasteur Institute, France). Anti-Flag M2 and anti- $\beta$-actin monoclonal antibodies were obtained from Sigma (St. Louis, MO, USA). Anti-Bax (N-20), anti-Bcl2 (N-19), anti-HA (Y-11), and anti-p73 (H-79) antibodies were purchased from Santa Cruz (CA, USA). Rabbit anti-cytochrome $c$ antibody was obtained from Cell Signaling (Danvers, MA, USA). Mouse anti-cytochrome $c$ antibody (Clone: 6H2.B4) was purchased from BD Pharmingen (MD, USA). AntiTimm50 antibody was purchased from Abcam (MA, USA). Secondary antibodies including Goat Anti-Mouse IgG and Goat Anti-Rabbit IgG were purchased from Jackson ImmunoResearch (West Grove, PA, USA).

Quantitative real time RT-PCR. Quantitative real-time PCR was performed with ABI PRISM 7700 Sequence Detection System Instrument and software (Applied Biosystems, Foster City, CA, USA), using the manufacturer's recommended conditions. Total RNA was isolated from transiently transfected cells (Trizol reagent, Invitrogen, CA), reverse transcribed (Superscript III, Invitrogen, $\mathrm{CA}$ ), and subjected to quantitative PCR analysis using Syber green master mix (Invitrogen, CA). The comparative threshold cycle (Ct) method was used to calculate the amplification factor, and the relative amount of target (TAp73) was normalized to GAPDH levels in parallel reactions. The primer sequences are as follows: Bax-forward $5^{\prime}$-CCGGCGAATTGGAGATGAACT-3' and reverse: $5^{\prime}$-CCAGCCCATGATGGTTCTGAT-3'. TAp73-forward 5'-GCGAGG AGTCCAACATGGAT-3' and reverse $5^{\prime}$-GGCACTGCTGAGCAAATTGA-3'. GAPDH-forward 5'-TGTGTCCGTCGTGCATCTGA- $3^{\prime}$ and reverse $5^{\prime}$-CCTGCT TCACCACCTTCTTGA-3'. P21-forward 5'-GAACTTTGACTTCGTCACGGAGA-3' and reverse 5'-CTCCGTTTTCGGCCCTGAGA-3'. Puma-forward 5'-CTCAGCC CTCCCTGTCACCA-3' and reverse $5^{\prime}$-GGGGAGGAGTCCCATGAAGAGA-3'

Immunofluorescence and DAPI staining. Cells were cultured on glass coverslips coated with $100 \mu \mathrm{g} / \mathrm{ml}$ poly-D-lysine. After fixing with $4 \%$ paraformaldehyde, and permeabilization with $0.2 \%$ Triton X-100, cells were stained with specific primary antibodies and with fluorescence-tagged secondary antibodies. Coverslips were mounted using Gel/Mount (Biomeda, CA) and observed by fluorescence or confocal microscopy (Olympus FV10i, Tokyo, Japan). Immunofluorescence for cytochrome $c$ in primary neurons was performed by briefly treating cells with $0.01 \%$ saponin ( 5 min on ice) as previously documented, ${ }^{22,48,49}$ which generates holes in the plasma membrane without permeabilization, leading to the 
release of cytosolic content. This was followed by fixation and normal immunostaining procedures.

\section{Conflict of Interest}

The authors declare no conflict of interest.

Acknowledgements. We thank Dr. Elisabetta Bianchi at the Pasteur Institute (France) for the RanBP9 monoclonal antibody. This work was supported in part by NIH/NIA (1R01AG033055-01A1, DEK), NIH/NIA (1K02AG031920-10A1, DEK), and WCU-Neurocytomics Project grant from NRF (DEK, HR).

1. Eckert A, Hauptmann S, Scherping I, Rhein V, Muller-Spahn F, Gotz J et al. Soluble betaamyloid leads to mitochondrial defects in amyloid precursor protein and tau transgenic mice. Neurodegener Dis 2008; 5: 157-159.

2. Manczak M, Anekonda TS, Henson E, Park BS, Quinn J, Reddy PH. Mitochondria are a direct site of $A$ beta accumulation in Alzheimer's disease neurons: implications for free radica generation and oxidative damage in disease progression. Hum Mol Genet2006; 15: 1437-1449.

3. Yao J, Irwin RW, Zhao L, Nilsen J, Hamilton RT, Brinton RD. Mitochondrial bioenergetic deficit precedes Alzheimer's pathology in female mouse model of Alzheimer's disease. Proc Natl Acad Sci USA 2009; 106: 14670-14675.

4. Li YP, Bushnell AF, Lee CM, Perlmutter LS, Wong SK. Beta-amyloid induces apoptosis in human-derived neurotypic SH-SY5Y cells. Brain Res 1996; 738: 196-204.

5. Loo DT, Copani A, Pike CJ, Whittemore ER, Walencewicz AJ, Cotman CW. Apoptosis is induced by beta-amyloid in cultured central nervous system neurons. Proc Natl Acad Sci USA 1993; 90: 7951-7955.

6. Kajkowski EM, Lo CF, Ning X, Walker S, Sofia HJ, Wang W et al. beta -Amyloid peptideinduced apoptosis regulated by a novel protein containing a g protein activation module. J Biol Chem 2001; 276: 18748-18756.

7. Dragicevic N, Mamcarz M, Zhu Y, Buzzeo R, Tan J, Arendash GW et al. Mitochondrial amyloid-beta levels are associated with the extent of mitochondrial dysfunction in different brain regions and the degree of cognitive impairment in Alzheimer's transgenic mice. J Alzheimers Dis 2010; 20(Suppl 2): S535-S550.

8. Reddy PH, Beal MF. Amyloid beta, mitochondrial dysfunction and synaptic damage: implications for cognitive decline in aging and Alzheimer's disease. Trends Mol Med 2008; 14: 45-53.

9. Abramov AY, Canevari L, Duchen MR. Beta-amyloid peptides induce mitochondria dysfunction and oxidative stress in astrocytes and death of neurons through activation of NADPH oxidase. J Neurosci 2004; 24: 565-575.

10. Du H, Guo L, Yan S, Sosunov AA, McKhann GM, Yan SS. Early deficits in synaptic mitochondria in an Alzheimer's disease mouse model. Proc Natl Acad Sci USA 2010; 107: 18670-18675.

11. Paradis E, Douillard H, Koutroumanis M, Goodyer C, LeBlanc A. Amyloid beta peptide of Alzheimer's disease downregulates Bcl-2 and upregulates bax expression in human neurons. J Neurosci 1996; 16: 7533-7539.

12. Kitamura $Y$, Shimohama S, Kamoshima W, Ota T, Matsuoka $Y$, Nomura $Y$ et al. Alteration of proteins regulating apoptosis, Bcl-2, Bcl-x, Bax, Bak, Bad, ICH-1 and CPP32, in Alzheimer's disease. Brain Res 1998; 780: 260-269.

13. Atabakhsh E, Bryce DM, Lefebvre KJ, Schild-Poulter C. RanBPM has proapoptotic activities that regulate cell death pathways in response to DNA damage. Mol Cancer Res 2009; 7: 1962-1972.

14. Coultas L, Huang DC, Adams JM, Strasser A. Pro-apoptotic BH3-only Bcl-2 family members in vertebrate model organisms suitable for genetic experimentation. Cell Death Differ 2002; 9: 1163-1166.

15. Letai A. BCL-2: found bound and drugged! Trends Mol Med 2005; 11: 442-444.

16. Andreyev AY, Kushnareva YE, Starkov AA. Mitochondrial metabolism of reactive oxygen species. Biochemistry (Mosc. ) 2005; 70: 200-214.

17. Marchi S, Giorgi C, Suski JM, Agnoletto C, Bononi A, Bonora M et al. Mitochondriaros crosstalk in the control of cell death and aging. J Signal Transduct 2012; 2012: 329635

18. Mayer B, Oberbauer R. Mitochondrial regulation of apoptosis. News Physiol Sci2003; 18: 89-94.

19. Lakshmana MK, Yoon IS, Chen E, Bianchi E, Koo EH, Kang DE. Novel role of RanBP9 in BACE1 processing of amyloid precursor protein and amyloid beta peptide generation. J Biol Chem 2009; 284: 11863-11872.

20. Lakshmana MK, Hayes CD, Bennett SP, Bianchi E, Reddy KM, Koo EH et al. Role of RanBP9 on amyloidogenic processing of APP and synaptic protein levels in the mouse brain. FASEB J 2012; 26: 2072-2083.

21. Lakshmana MK, Chung JY, Wickramarachchi S, Tak E, Bianchi E, Koo EH et al. A fragment of the scaffolding protein RanBP9 is increased in Alzheimer's disease brains and strongly potentiates amyloid-beta peptide generation. FASEB J 2012; 24: 119-127.

22. Woo JA, Roh SE, Lakshmana MK, Kang DE. Pivotal role of RanBP9 in integrin-dependent focal adhesion signaling and assembly. FASEB J 2012; 26: 1672-1681.

23. Woo JA, Jung AR, Lakshmana MK, Bedrossian A, Lim Y, Bu JH et al. Pivotal role of the RanBP9cofilin pathway in Abeta-induced apoptosis and neurodegeneration. Cell Death Differ 2012; 19: 1413-1423.

24. Kang DE, Roh SE, Woo JA, Liu T, Bu JH, Jung AR et al. The interface between cytoskeleta aberrations and mitochondrial dysfunction in Alzheimer's disease and related disorders. Exp Neurobiol 2011; 20: 67-80.
25. Kramer S, Ozaki T, Miyazaki K, Kato C, Hanamoto T, Nakagawara A. Protein stability and function of p73 are modulated by a physical interaction with RanBPM in mammalian cultured cells. Oncogene 2005; 24: 938-944.

26. John K, Alla V, Meier C, Putzer BM. GRAMD4 mimics p53 and mediates the apoptotic function of p73 at mitochondria. Cell Death Differ 2011; 18: 874-886.

27. Lambert AJ, Brand MD. Reactive oxygen species production by mitochondria. Methods Mol Biol 2009; 554: 165-181.

28. Frank S, Gaume B, Bergmann-Leitner ES, Leitner WW, Robert EG, Catez F et al. The role of dynamin-related protein 1, a mediator of mitochondrial fission, in apoptosis. Dev Cell 2001; 1: 515-525.

29. Smirnova E, Griparic L, Shurland DL, van der Bliek AM. Dynamin-related protein Drp1 is required for mitochondrial division in mammalian cells. Mol Biol Cell 2001: 12: 2245-2256.

30. Eiben LJ, Duckett CS. The IAP family of apoptotic regulators. Results Probl Cell Differ 1998; 24: 91-104.

31. Janumyan YM, Sansam CG, Chattopadhyay A, Cheng N, Soucie EL, Penn LZ et al. Bcl-xL/ $\mathrm{Bcl}-2$ coordinately regulates apoptosis, cell cycle arrest and cell cycle entry. EMBO J 2003; 22: $5459-5470$.

32. Sayan AE, Sayan BS, Gogvadze V, Dinsdale D, Nyman U, Hansen TM et al. P73 and caspase-cleaved p73 fragments localize to mitochondria and augment TRAIL-induced apoptosis. Oncogene 2008; 27: 4363-4372.

33. Chang LK, Liu ST, Kuo CW, Wang WH, Chuang JY, Bianchi E et al. Enhancement of transactivation activity of Rta of Epstein-Barr virus by RanBPM. J Mol Biol 2008; 379: 231-242.

34. Poirier MB, Laflamme L, Langlois MF. Identification and characterization of RanBPM, a novel coactivator of thyroid hormone receptors. J Mol Endocrinol 2006; 36: 313-325.

35. Rao MA, Cheng H, Quayle AN, Nishitani H, Nelson CC, Rennie PS. RanBPM, a nuclear protein that interacts with and regulates transcriptional activity of androgen receptor and glucocorticoid receptor. J Biol Chem 2002; 277: 48020-48027.

36. Bernstein BW, Bamburg JR. ADF/cofilin: a functional node in cell biology. Trends Cell Biol 2010; 20: 187-195.

37. Chua BT, Volbracht C, Tan KO, Li R, Yu VC, LiP. Mitochondrial translocation of cofilin is an early step in apoptosis induction. Nat Cell Biol 2003; 5: 1083-1089.

38. Klamt F, Zdanov S, Levine RL, Pariser A, Zhang Y, Zhang B et al. Oxidant-induced apoptosis is mediated by oxidation of the actin-regulatory protein cofilin. Nat Cell Biol 2009; 11: $1241-1246$

39. Wabnitz GH, Goursot C, Jahraus B, Kirchgessner H, Hellwig A, Klemke M et al. Mitochondrial translocation of oxidized cofilin induces caspase-independent necrotic-like programmed cell death of T cells. Cell Death Dis 2010; 1: e58.

40. Wetzel MK, Naska S, Laliberte CL, Rymar VV, Fujitani M, Biernaskie JA et al.p73 regulates neurodegeneration and phospho-tau accumulation during aging and Alzheimer's disease. Neuron 2008; 59: 708-721.

41. Pozniak CD, Radinovic S, Yang A, McKeon F, Kaplan DR, Miller FD. An anti-apoptotic role for the p53 family member, p73, during developmental neuron death. Science 2000; 289 : 304-306.

42. Cabrera-Socorro A, Pueyo MM, Suarez Sola ML, Gonzalez Delgado FJ, CastaneyraPerdomo A, Marin MC et al. Multiple isoforms of the tumor protein p73 are expressed in the adult human telencephalon and choroid plexus and present in the cerebrospinal fluid. Eur J Neurosci 2006; 23: 2109-2118.

43. Wilson C, Henry S, Smith MA, Bowser R. The p53 homologue p73 accumulates in the nucleus and localizes to neurites and neurofibrillary tangles in Alzheimer disease brain. Neuropathol Appl Neurobiol 2004; 30: 19-29.

44. Hooper C, Killick R, Tavassoli M, Melino G, Lovestone S. TAp73alpha induces tau phosphorylation in HEK293a cells via a transcription-dependent mechanism. Neurosci Let 2006; 401: 30-34.

45. Cancino GI, Miller FD, Kaplan DR. p73 haploinsufficiency causes tau hyperphosphorylation and tau kinase dysregulation in mouse models of aging and Alzheimer's disease. Neurobiol Aging 2013; 34: 387-399.

46. Zhang $\mathrm{H}$, Wu S, Xing D. YAP accelerates Abeta(25-35)-induced apoptosis through upregulation of Bax expression by interaction with p73. Apoptosis 2011; 16: 808-821.

47. Alvarez AR, Sandoval PC, Leal NR, Castro PU, Kosik KS. Activation of the neuronal c-Abl tyrosine kinase by amyloid-beta-peptide and reactive oxygen species. Neurobiol Dis 2004; 17: 326-336.

48. Kang DE, Soriano S, Xia X, Eberhart CG, De SB, Zheng H et al. Presenilin couples the paired phosphorylation of beta-catenin independent of axin: implications for beta-catenin activation in tumorigenesis. Cell 2002; 110: 751-762.

49. Soriano S, Kang DE, Fu M, Pestell R, Chevallier N, Zheng H et al. Presenilin 1 negatively regulates beta-catenin/T cell factor/lymphoid enhancer factor-1 signaling independently of beta-amyloid precursor protein and notch processing. J Cell Biol 2001; 152: 785-794.

Cell Death and Disease is an open-access journal published by Nature Publishing Group. This work is licensed under the Creative Commons Attribution-NonCommercial-No Derivative Works 3.0 Unported License. To view a copy of this license, visit http://creativecommons.org/licenses/by-nc-nd/3.0/ 